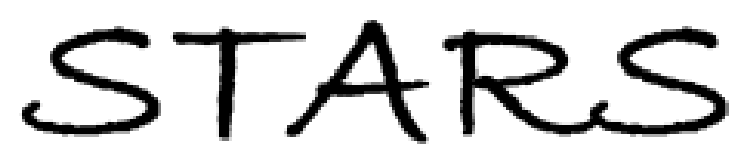

University of Central Florida

STARS

$1-1-2002$

\title{
VE-based training strategies for acquiring survey knowledge
}

Bob G. Witmer

Wallace J. Sadowski

University of Central Florida

Neal M. Finkelstein

Find similar works at: https://stars.library.ucf.edu/facultybib2000

University of Central Florida Libraries http://library.ucf.edu

This Article is brought to you for free and open access by the Faculty Bibliography at STARS. It has been accepted for inclusion in Faculty Bibliography 2000s by an authorized administrator of STARS. For more information, please contactSTARS@ucf.edu.

\section{Recommended Citation}

Witmer, Bob G.; Sadowski, Wallace J.; and Finkelstein, Neal M., "VE-based training strategies for acquiring survey knowledge" (2002). Faculty Bibliography 2000s. 3550.

https://stars.library.ucf.edu/facultybib2000/3550

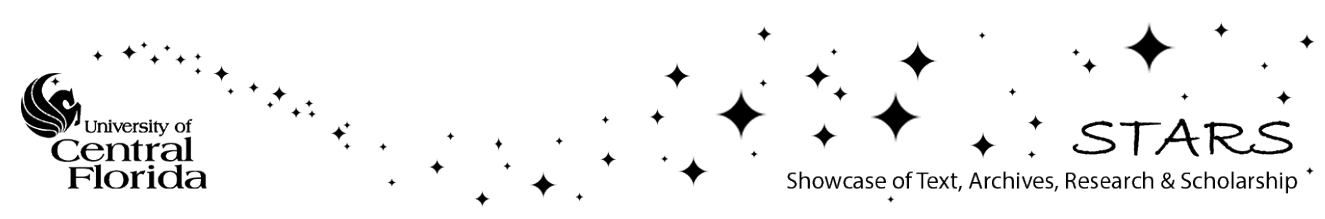




\section{Bob G. Witmer}

Bob_Witmer@stricom.army.mil U.S. Army Research Institute for the Behavioral and Social Sciences Simulator Systems Research Unit

\section{Wallace J. Sadowski}

University of Central Florida

\section{Neal M. Finkelstein}

U.S. Army Simulation, Training, and Instrumentation Command
Presence, Vol. I I, No. I, February 2002, 1-18 (c) 2002 by the Massachusetts Institute of Technology

\section{VE-Based Training Strategies for Acquiring Survey Knowledge}

\begin{abstract}
Virtual environments (VEs) have been used successfully to train wayfinders to navigate through buildings and learn their layout. However, at the same time, for many, the VE deficiencies have reduced the effectiveness of VEs for training spatial tasks. In an effort to improve $V E$ effectiveness, we conducted research to determine if certain unique capabilities of VEs could compensate for its deficiencies. Research participants were required to learn the layout or configuration of one floor of an office building as portrayed in a VE. To improve spatial learning, we developed three VE navigation training aids: local and global orientation cues, aerial views, and a themed environment enhanced with sights and sounds and divided into four distinct sectors. The navigation aids were provided during the training but were not available during testing of survey knowledge. Of the three training aids investigated, only the aerial views were effective in improving performance on the survey knowledge tests. The effectiveness of the navigation aids seemed to depend on how they were used during training. A retention test given one week after training indicated that spatial knowledge acquired in a VE diminished little over the one-week retention interval.
\end{abstract}

\section{Background}

For nearly ten years, the U.S. Army Research Institute (ARI) for the Behavioral and Social Sciences has conducted a research program to investigate the utility of virtual environments (VEs) for training skills needed by dismounted soldiers. Much of that research has involved creating VEs that were as similar to their real-world counterparts as possible, in order to replicate realworld training and to maximize skill transfer. Our attempts to replicate realworld training uncovered deficiencies in VE technology that reduced its training utility (Witmer, Bailey \& Knerr, 1995; Witmer, Bailey, Knerr, \& Parsons, 1996). When training in VEs, some trainees experienced simulator sickness, became lost or disoriented, or manifested perceptual distortions. However, VEs also possess unique characteristics that make them more flexible training media and that may enhance training effectiveness. In VEs, multiple modes of locomotion are available, movement speed can be much faster than in the real world, and participants can be instantly transported from one location to another. VEs also permit viewing from multiple perspectives and can be augmented easily with visual and auditory features that aid learning. The purpose of this paper is to examine the cognitive and perceptual variables that are thought to influence the speed and overall quality of spatial knowledge acquisition by using the unique possibilities provided by an immersive VE. 


\section{Spatial Knowledge Acquisition}

Much research has been conducted to understand how humans and animals acquire information about the spaces that surround them and how they are able to navigate complex environments. More than fifty years ago, Tolman (1948) suggested that his rats were using tentative cognitive maps indicating routes and paths to a goal location. Although controversial at the time, Tolman's idea that a cognitive map guides mammalian spatial behavior has gained wide acceptance. Levine (1951) suggested that, as people move through space, they divide it into regions, paths, and barriers because space, in a philosophical sense, is empty and needs bounding and identification. The distinction between paths and bounded regions forms the basis for two different types of spatial knowledge: route knowledge and survey knowledge.

\section{I Routes, Landmarks, and Survey Knowledge}

Siegel and White (1975) distinguished route knowledge (a sequential record of the space between the start point, landmarks along the route, and the destination) and survey knowledge (an awareness of the topographic properties of the environment). Survey knowledge is considered present when (i) significant portions of the spatial layout are memorized allowing for calculation of distances between landmarks and destinations (Euclidean and route), (ii) directions to destinations can be indicated accurately, and (iii) shortcuts can be engineered. As a person acquires survey knowledge, a cognitive map or similar representation develops and is embedded in long-term memory (Golledge, 1987). This allows the use of mental imagery to determine direction to designated locations, estimate distances, and determine appropriate routes; it also enables individuals to infer spatial relationships that were not directly trained or experienced.

The current study combines three measurement approaches that encapsulate the defining characteristics of survey knowledge just described. Participants demonstrate knowledge of the spatial layout by completing a map construction task, and knowledge of distance and directions between landmarks by directly estimating heading and distance. Finally, they show that they can engineer shortcuts by first training on lobby-to-landmark spatial relations and then by inferring the shortest route between pairs of landmarks.

\subsection{Methods of Acquiring Spatial Knowledge}

Generally, spatial knowledge is learned from maps or by navigating the environment. Floor plans, blueprints, and "you-are-here" maps are commonly used maps that provide a vertical view from the outside looking in (that is, an exocentric perspective). Navigating an environment provides a horizontal view from within the environment (an egocentric perspective). Thorndyke and Hayes-Roth (1982) found that navigating an environment is the best way to obtain knowledge of routes and landmarks. Maps were best for rapidly acquiring survey knowledge, but, with extended navigation of the environment, navigation-trained survey knowledge exceeded map-trained survey knowledge.

\subsection{Measuring Survey Knowledge}

The traditional way to measure survey knowledge is to require learners to sketch a map of the layout (Lynch, 1960; Appleyard, 1970). Although map sketches have high face validity and clearly demonstrate knowledge acquisition, sketches are difficult to score and may underestimate how much knowledge has been acquired. Wickens and Prevett (1995) used an easily scored variation of the map-sketching task, asking participants to arrange paper cutouts of the objects in an environment on a piece of paper to re-create their layout in the previously experienced environment. This map reconstruction task was scored by measuring the linear distance from each participant's placement of a terrain feature to the actual location of the feature in the environment. Variations of the map reconstruction task have been used successfully by other researchers to measure survey knowledge (Goerger et al., 1998; Waller, 1999). 
Another way to assess survey learning is to require participants to determine the direction (or bearing) and distance to unseen target locations. This is most commonly done by asking the participant to turn toward the unseen goal location and to verbally report the distance to the goal (Siegel, 1981). Either absolute or relative error measures can be computed. Absolute measures include heading error and distance error. Heading error is the absolute value of the angular difference between the estimated direction and the true target direction; distance error is the absolute value of the difference between the estimated and true target distances. Relative measures of distance estimates include the correlation between the estimated and actual distances (Waller, 1999) and relative distance error (Witmer \& Kline, 1997).

Another method requires that participants not merely know the layout but also be able to apply that knowledge by following the most direct route between two designated landmarks or destinations (Witmer et al., 1995; Satalich, 1995). The most direct route test involves routes that have not been specifically trained and that may never have been previously traversed. This method is based on the notion that survey knowledge might be a necessary condition for the invention of new routes (Siegel \& White, 1975). Of the measurement methods used in this research, the most direct route test is perhaps the most appealing from an applied standpoint because it forces participants to put their survey knowledge into practice.

\subsection{Acquiring Survey Knowledge in VEs}

Witmer et al. (1996) used a VE model of a complex office building to demonstrate transfer of route knowledge, but not survey knowledge, to the real building. Witmer et al. suggested that survey knowledge might not have been acquired because no special effort was made to convey that knowledge directly; rather, it was expected that survey knowledge would be acquired incidentally while learning the route. Although participants studied a large, detailed, paper-based route map during the initial $15 \mathrm{~min}$. training phase, instructions directed participants to learn the route, not the layout.
Witmer et al. also identified problems associated with the VE that may have interfered with survey learning; these included a reduced field of view, disorientation following collisions with virtual walls, and simulator sickness.

Contrary to expectations, Witmer et al. found that studying a paper map prior to VE exposure had no effects on the acquisition of route or survey knowledge in the VE. Satalich (1995), on the other hand, found that a control group that received only a map outperformed groups that had direct exposure to the VE on a variety of measures of route and survey knowledge. Although the VE modeled for Satalich's study was by no means simple, consisting of 39 separate rooms and more than five hundred objects, Satalich indicated that it was organized in a fashion to enhance survey learning. Goerger et al. (1998) found that a group that studied a map of a complex building performed significantly better on one of three measures of survey knowledge than a VE rehearsal group that also had a map. Miller, Clawson, and Sebrechts (1999) showed that a VE-trained group did not perform as well as a map-trained group on a pointing measure of survey knowledge after a two-week retention interval. The relative effectiveness of VE versus maps for acquiring spatial knowledge depends on many factors, including the complexity of the environment, the exposure time to the VE or maps, the nature of the spatial tasks, and the quality of the VE and maps, to name a few.

More recently, researchers have demonstrated that survey knowledge can be acquired in VEs. Ruddle, Payne, and Jones (1997) demonstrated increased survey knowledge after nine unguided sessions in a VE. Wilson, Foreman, and Tlauka (1997) showed that participants trained to locate objects in a VE were more accurate in a real-world pointing task than were controls who had no previous exposure to the VE. They also found that participants trained in a virtual building outperformed controls in drawing the building layout, but did not differ from participants trained in the real building. Wilson et al. (1997) attributed their success in demonstrating transfer of survey knowledge from VE to the real world to using a simpler building layout than was used by Witmer et al. (1996). Koh, von Wiegand, 
Garnett, Durlach, and Shinn-Cunningham (1999), using a relatively simple building layout, demonstrated that survey knowledge acquired in a virtual building transferred to the real world and was comparable to the same knowledge acquired in the actual building. Gillner and Mallot (1997) showed that survey knowledge as represented by drawings of the perceived layout could be acquired in a VE. The layout consisted of repeating Y-shaped junctions (with three landmarks unique to each) linked together by the extension of one leg of the Y. Gillner and Mallot's work demonstrated that simple configurations could be acquired in a VE in a reasonable time without the use of maps.

Advantages of using an immersive VE for training survey knowledge include four-dimensional representations of the environment (including time), ease of adding visual and auditory enhancements to aid learning, the ability to have multiple perspectives and modes of locomotion, and access to learning environments that may be otherwise unavailable. With VE, there are fewer constraints on training access, real-world hazardous environments can be represented without endangering personnel, and trainees develop the confidence and familiarity that comes from the sense of being "present" in the environment without having to travel to distant real-world sites for training. VEs can be indispensable for rehearsing military missions, particularly those involving search and rescue, or other operations in unfamiliar or hostile environments.

\subsection{Designing Spaces that Enhance Spatial Knowledge Acquisition}

Lynch (1960) identified design elements of urban spaces that people used to define and organize those spaces. He found regularities in the types of environmental features that were most often noticed, and hence, remembered. Lynch found that landmarks that contrast with their surroundings and landmarks that could be seen from many different locations were more frequently noticed. Landmarks that have directional information associated with them were reported to help people orient themselves. Lynch noted that cities often have identifiable bounded districts that differ in form or function. The age of structures or architectural style may set a district apart from neighboring districts, or the district may be defined according to its function (such as an industrial district). Distinguishing features of city districts are not limited to visual appearance; districts may have distinguishing sounds or smells. Darken and Sibert (1996), drawing on Lynch's work, developed the following organizational principles for supporting wayfinding tasks in VE and in the real world.

- Divide the larger environment into smaller identifiable parts (or districts) to preserve a sense of place.

- Organize the smaller parts under a simple organizational principle, such as a grid or other logical spatial ordering.

- Provide frequent directional cues to maintain orientation in the environment and preclude the wayfinder from becoming disoriented.

In an exploratory study, Darken and Sibert (1993) investigated the use of various navigation aids (such as a virtual sun, breadcrumbs, and flying) in locating a target during VE exploration and in returning "home" to the start point. Although they presented no quantitative data, they reported that the navigation aids exerted a strong influence on navigation strategies and performance. For example, they found that the inclusion of a single fixed feature (such as a virtual sun) that could be seen from any vantage point within the environment improved performance on a search task in a VE. They attributed the effectiveness of the virtual sun to two characteristics: its relative immobility and its visibility throughout the environment. In the present study, a similar navigation aid (a virtual flagpole) served as a global reference point for orienting within the VE during the training phase. This global cue was coupled with the local orientation cue, described in the next paragraph, to help participants maintain their orientation in the VE.

Witmer et al. (1996) reported that collisions with walls in the VE and the subsequent disorientation might have interfered with the acquisition of spatial knowledge. To reduce these effects in the present study, the VE used in the 1996 study was upgraded to reduce collisions (hallways and doorways were widened) and a lo- 
cal orienting aid was provided. The local orienting aid consisted of an arrow projecting from the chest of the participant's avatar to indicate the current direction of travel. This device reduces collisions by allowing participants to know their precise heading relative to objects in the VE, irrespective of head movement.

As discussed by Lynch (1960) and reiterated by Darken and Sibert (1993), we divided the VE into four distinct districts or quadrants to promote spatial knowledge acquisition. Each quadrant was populated with virtual objects (or landmarks) representing a particular theme. Although a quadrant's theme was apparent throughout the quadrant, the most salient themed landmarks were reserved for destination rooms. To make these landmarks more memorable, destination landmarks had both visual and auditory aspects. To ensure that participants associated the themed landmarks with the destinations, participants were forced to interact with these landmarks by identifying the theme represented. A similar technique was shown to increase recall of landmark location previously (Guest, Bliss, \& Lohmeier, 1997; Guest \& Sadowski, 1997).

Some of our research participants were permitted aerial views of the environment (exocentric view), then returned to a normal viewing perspective (egocentric view). Viewing in which participants can switch between egocentric and exocentric views has been termed bicentric viewing (Salzman, Dede, Loftin, \& Ash, 1998). The aerial views provided a map-like view of the entire environment at the maximum height, or some portion of the environment at lesser viewing heights. At the lesser heights, an aerial view (in contrast to most maps) allows direct viewing of landmarks. The value of using maps for acquiring spatial knowledge in VEs has been well documented (Satalich, 1995; Darken \& Sibert, 1996; Bliss, Tidwell, \& Guest, 1997; Miller et al., 1999), but the effects on spatial learning of using a bird's-eye perspective for viewing virtual spaces has rarely been investigated. Darken and Sibert (1993) reported that providing a bird's-eye view of an outside environment can aid efficient navigation. More recently, Koh et al. (1999) employed a virtual miniature 3-D model of one floor of a building displayed as an aerial view that could be manipulated using a mouse and a monitor. They showed that this model was as good as a VE or a real-world environment for acquiring survey knowledge.

In the current research, we investigated three enhancements to VE in aiding survey learning: global and local orientation aids, themed quadrants or districts, and aerial views. By adding these enhancements to the basic VE, we have purposely made the VE less like the real world, but the enhancements should also aid navigation through the VE and allow participants to more easily structure the VE for increased memorability.

It should be noted that the type of VE enhancements that we used would be impractical or impossible to implement in an existing real-world interior environment. A bird's-eye view of interior spaces requires removing the ceiling, as does the inclusion of a virtual flagpole. The local orientation arrow, although possible in the real world, is not needed in that environment because real-world collisions are rare. The themed quadrants that were superimposed on the basic VE would be extremely difficult to implement in the real world, even if practical considerations did not completely rule out such disruptive alterations to the real environment.

\section{VE Navigation Aids Experiment}

\section{I Method}

3.I.I Participants. Students attending a local university participated in this research. The participants-32 men and 32 women-ranged from 18 to 47 years of age, with a mean age of 23.08 years. Forty-four of these participants had no previous VE experience. All participants had normal vision uncorrected or corrected with contact lenses or glasses. Participants were screened for any history of seizures or other physical problems that would preclude their participation in this research. They were also screened for prior exposure to or knowledge of the building layout represented in the VE. They received either a cash payment or extra class credit for participating in the research.

3.I.2 Equipment and Materials. A computer model of one floor of a large office building (used in previous research by Bailey and Witmer (1994) and 


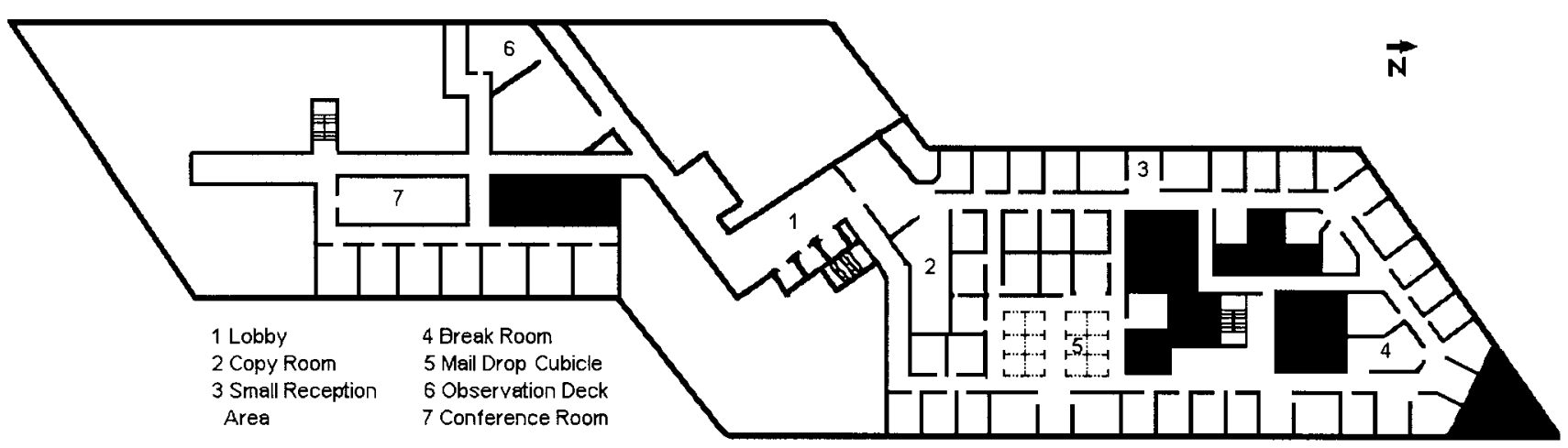

Figure I. Layout of VE model of one floor of a large office building showing the location of destination rooms.

Witmer et al. (1996)) was adapted for this experiment. The 64-room VE model encompassed approximately 4,088 square meters and was created using more than 35,000 polygons. A schematic of the model is shown in figure 1. Separate VE models were constructed to represent the standard and the enhanced environments. The enhanced environment model was created by adding theme objects and sounds to the standard environment model.

The VEs were modeled using Multigen II software and rendered by a Silicon Graphics Onyx with eight 200 $\mathrm{MHz}$ processors and three RealityEngine2 graphics pipes. Both models were displayed using a Virtual Research V8 helmet-mounted display (HMD). The V8 provides a color, high-resolution $(640(\mathrm{x} 3) \times 480) 3-\mathrm{D}$ view of the VE, but has a limited field of view (FOV) of $48 \mathrm{deg}$. horizontal and $36 \mathrm{deg}$. vertical. Localized, directional sound was provided in the enhanced environment through the HMD headphones. Custom software developed by the Institute for Simulation and Training (IST) controlled the presentation of trials and automatically recorded the data.

A raised circular platform with a safety rail allowed for simulated walking. (See figure 2.) The platform, developed by IST, has been used successfully in previous experiments (Singer, Ehrlich, \& Allen, 1998; Lampton \& Parsons, 2001). A lightweight backpack worn by the participant provided connections to six position sensors,

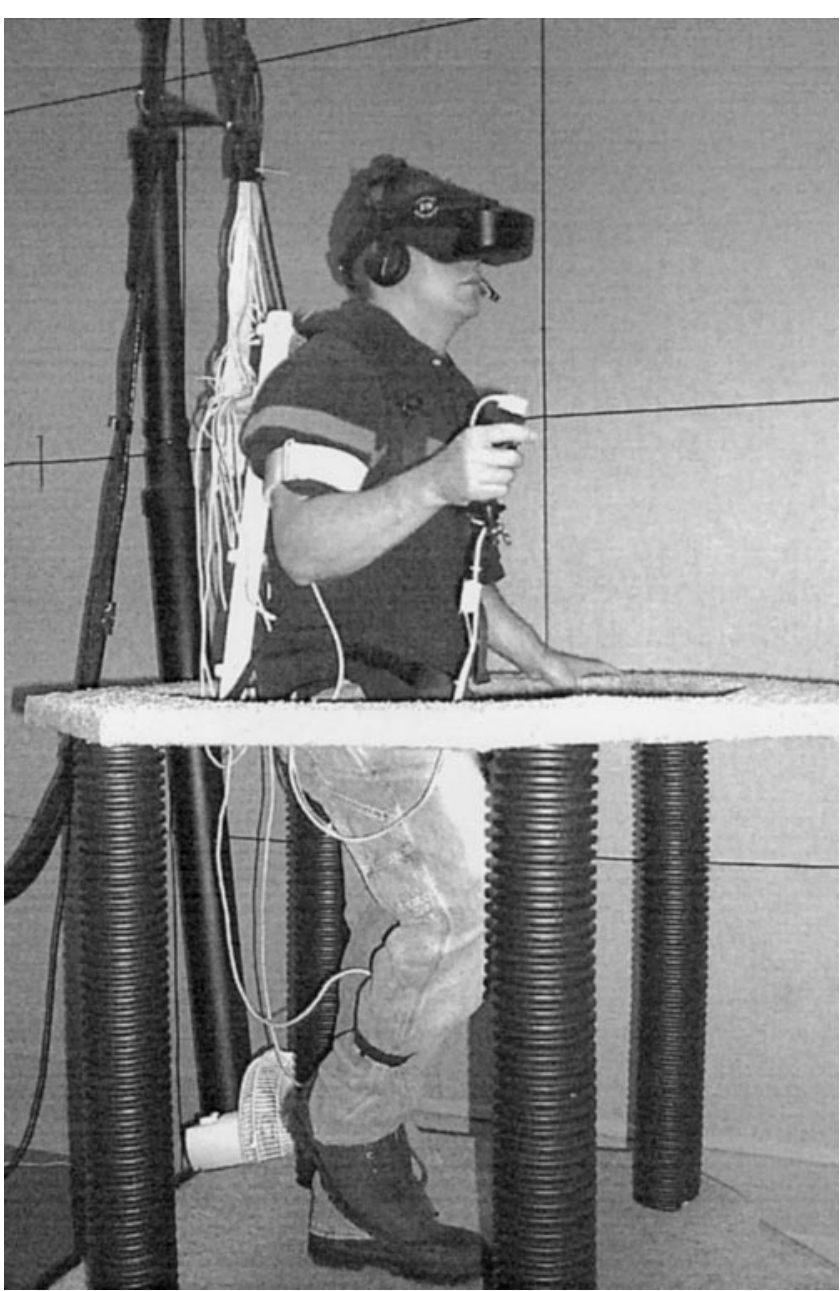

Figure 2. Walking platform and interface including a VE display sensor and a hand controller sensor. A sensor was also attached to the right arm just 
above the elbow, another on the backpack, and one on each ankle. Movements were accurately tracked by an Ascension MotionStar. The position sensors allowed participants to walk in place and independently turn their head and body in any direction. The hand controller was used to set an initial reference point for measuring angular head and body displacement and for controlling aerial viewing height for participants with access to an aerial view.

Participants had virtual representations of their body in the form of gender-specific avatars. Movements of the participants' head, torso, and right arm were simulated in the avatar's movements; however, leg movements were not articulated and produced only translational movement of the avatar. Although participants could see some parts of their avatar if they tried; their avatars were generally not seen, except for participants who had an aerial view. All participants could see the tour guide avatar, however.

Four questionnaires were used: two for measuring immersion, a third for measuring comfort (simulator sickness), and a fourth for collecting subject demographics. The Presence Questionnaire (PQ) and Immersive Tendencies Questionnaire (ITQ) were used for measuring immersion (Witmer \& Singer, 1998) and the Simulator Sickness Questionnaire (SSQ) (Kennedy, Lane, Berbaum, \& Lilienthal, 1993) was used to measure simulator sickness. The cubes comparison test, from the Educational Testing Service Kit of Factor-Referenced Cognitive Tests (Ekstrom, French, Harman, \& Dermen, 1990) measured each individual's spatial ability. The cube comparison test requires participants to examine a cube with letters, numbers, and shapes/figures on its faces to determine whether it is the same as a comparison cube. Participants must complete 42 items on the test in $6 \mathrm{~min}$. or less. The test items may be completed using various strategies including mental rotation of the cubes, but mental rotation is not required for every item (Just \& Carpenter, 1985).

3.1.3 Procedure. A researcher briefed participants regarding the purpose of the research, warned them about the potential for simulator sickness, and provided an overview of the tasks to be performed. Par- ticipants were assigned randomly to one of eight treatment groups with the restriction that each group comprised equal numbers of men and women. The experiment represents a 2 (presence/absence of orientation cues $) \times 2$ (presence/absence of aerial perspective $)$ $\times 2$ (presence/absence of themes) $\times 2$ (male $/$ female gender) between-subjects design.

After being briefed on the procedure and completing the cube comparisons test, SSQ, ITQ, and demographic surveys, participants practiced moving through a series of rooms connected by narrow passageways in the VE to introduce them to the equipment and to familiarize them with VE movement techniques. They also practiced turning in place and backing away from objects. Participants in the aerial groups used the hand controller to transition to and from an aerial perspective and practiced moving while airborne. Participants remained in the practice VE for a maximum of $10 \mathrm{~min}$. followed by a $5 \mathrm{~min}$. break.

Participants then received instructions specific to their treatment group. Aerial groups could view the VE from heights of 15, 30, and $120 \mathrm{~m}$ (exocentric perspective) for a period of up to $1 \mathrm{~min}$. After $1 \mathrm{~min}$., they automatically returned to the normal (egocentric) perspective view. They could choose aerial views one time or many times, but the viewing period was limited to $1 \mathrm{~min}$. each time. The $1 \mathrm{~min}$. limit was imposed so that participants had adequate ground time to visit all destination rooms during the time-limited training phases. Except for the l min. limit on continuing aerial views, individual participants determined how much time they spent in the aerial view during free exploration and during search training. Viewing heights were selected such that participants could see either the whole third-floor layout simultaneously at $120 \mathrm{~m}$, or parts of the layout at 15 and $30 \mathrm{~m}$. At a viewing height of $15 \mathrm{~m}$, about one-eighth of the environment was visible in a single glance, but objects within the environment could be recognized easily. At $30 \mathrm{~m}$, one-fourth of the environment was simultaneously visible, but objects were more difficult to recognize. Figure 3 shows a view of the enhanced VE from a height of $30 \mathrm{~m}$. When aerial group participants initially pressed the trigger button on their hand controller, they gradually ascended to $15 \mathrm{~m}$. They could increase view- 


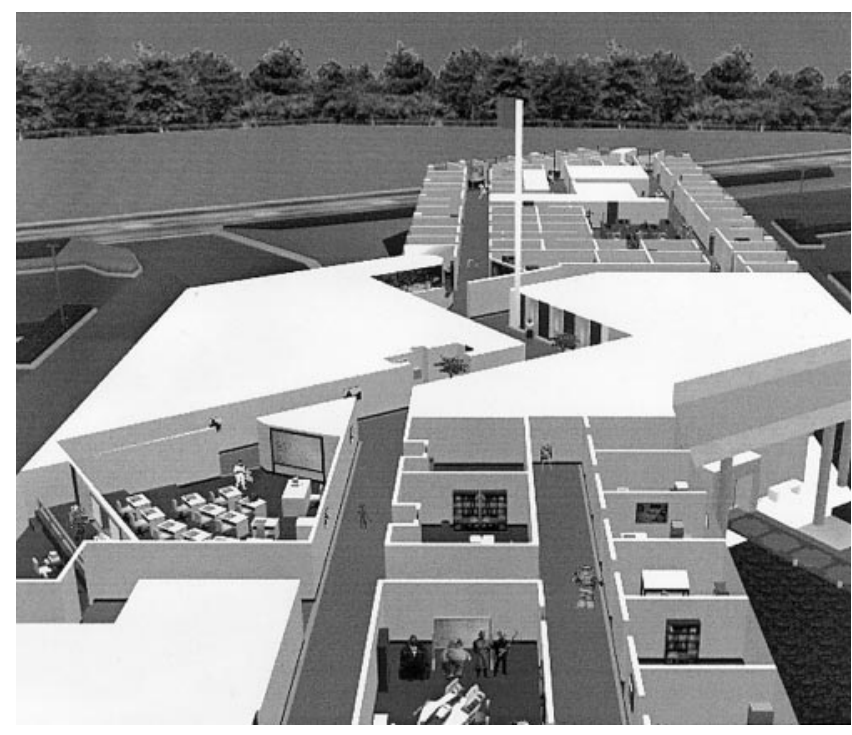

Figure 3. Enhanced VE at $30 \mathrm{~m}$ viewing height.

ing height by pressing the trigger button a second or third time. While in the air, participants could further explore the environment by flying to other locations (accomplished by walking in place). To return to ground level, they pressed the thumb button on their hand controller, and gradually descended to reenter their virtual body at the exact location where they left it when they started to fly. To minimize neck strain while flying, their view was pitched $30 \mathrm{deg}$. downward from the horizontal plane when facing straight ahead. Of course, participants could alter the pitch angle by moving their head.

Groups exposed to the themed environment encountered sights and sounds associated with each of four themed quadrants. Inside each destination room was a memorable theme object and an associated sound that became louder as the participant approached the destination. The addition of sound was expected to make the destinations easier to find during training and to increase the strength of association between the destination rooms and their respective quadrants for better recall during testing. Figure 4 shows the normal perspective view of one destination room enhanced with a sports theme object. Additional theme objects were positioned along the building corridors, but no sounds

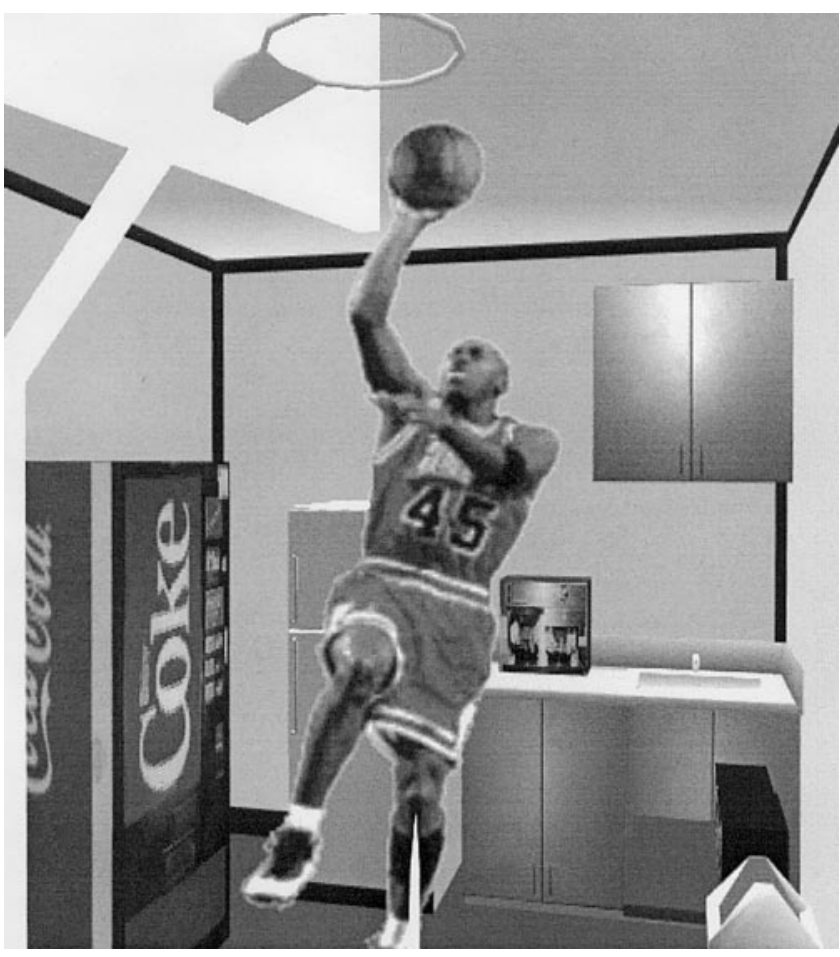

Figure 4. Normal perspective view of a destination room enhanced with a sports theme object.

were associated with them. The themes embedded in the quadrants were a tropical islands theme, a wild animals theme, an extraterrestrial (or outerspace) theme, and a sports theme. Upon encountering a theme object inside a destination room, participants were asked to identify the theme represented by that object. Hypothetically, participants could use this information to organize the building into four distinctive sectors and associate destinations with their respective sectors.

The orientation cue groups were asked to relate their current position to their starting position (in the lobby) marked by a virtual flagpole. This was accomplished by having participants turn and face the flagpole upon reaching each destination. The flagpole, visible throughout the environment, served as a global orientation cue that permitted participants to update their position continuously based on their known starting position. In addition to this global orientation cue, participants were instructed to use the arrow projecting from the chest of 


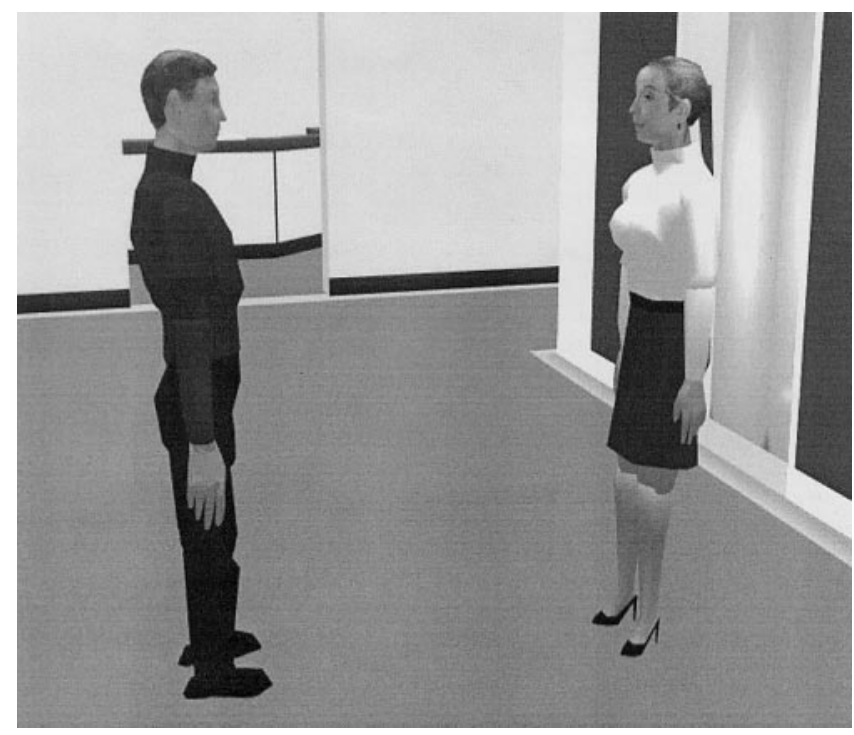

Figure 5. Female avatar tour guide and male avatar representing the participant.

their avatar as an indication of their current heading and as a way of aligning their virtual body so as to avoid collisions with walls and doorways.

The training was conducted in three distinct phases. During the first phase (guided tour), all participants took a guided tour of the VE, pausing at each destination room, and identifying it by name. Participants actively followed a virtual tour guide to each destination. The virtual tour guide described the "non-theme related" distinguishing features of each destination along the way. Figure 5 illustrates a female tour guide avatar and an avatar representing a male participant. The gender of the tour guide was always opposite the participant's gender. In the second phase (free exploration), participants explored the VE freely for $10 \mathrm{~min}$., while trying to accomplish the assigned goal of locating and identifying each previously visited destination. In the final phase (destination search), participants attempted to take the shortest route from the third-floor lobby to each named destination. If the participants did not find the destination within $3 \mathrm{~min}$., they were verbally guided to it.

Following this training, survey knowledge acquisition was measured by administering three separate tests in the following order: The projective convergence test, the most direct route test, and the room placement test. The room placement test was administered a second time approximately one week after the initial administration to assess retention of survey knowledge acquired in a VE. Only 59 of 64 participants ( 31 men and 28 women) completed the retention test. Because participants did not know in advance what they would be required to do, it was unlikely that they made any special effort to memorize their room placement when tested initially or that any rehearsal occurred during the retention interval.

The twelve-item projective convergence test required participants to indicate the direction and distance to four destination rooms from each of three different sighting locations. Participants were instructed to turn toward and walk to the center of the destination room. They indicated a destination's direction by facing the destination, and the perceived distance to the destination by attempting to walk to the destination without vision. Previous research (Witmer \& Sadowski, 1998) found that distance estimates produced by this method are more accurate than verbal distance estimates, both in the real world and in VEs. The minimum and maximum distances between the sighting locations and the destination rooms were $15.76 \mathrm{~m}$ and $89.87 \mathrm{~m}$, respectively. The measures selected for this test were heading error to assess the accuracy of bearing estimates and distance error to assess the accuracy of estimated distance. The bearing error was the absolute value of the angular divergence (in degrees) of the estimated location from the actual location. The distance error was the absolute value of the difference between the estimated and actual distances in meters.

The most direct route test required participants in the VE to determine the shortest route between two named locations. All participants completed this task in the same order for six destination pairs. Pairing of the destinations and testing order were selected to ensure that the test adequately measured survey knowledge rather than knowledge of previously traversed routes. Test measures included the mean distance error in locating the destinations (the mean of the differences between the shortest distance to the destination and the distance 
Table I. Untransformed Means and Standard Deviations for Training Phase Performance Measures as a Function of Type of Navigation Aid

\begin{tabular}{lllll}
\hline $\begin{array}{l}\text { Navigation } \\
\text { Aid Provided }\end{array}$ & $\begin{array}{l}\text { Rooms Found: } \\
\text { Exploration Phase }\end{array}$ & $\begin{array}{l}\text { Rooms Found: } \\
\text { Search Phase }\end{array}$ & $\begin{array}{l}\text { Search Time: } \\
\text { Search Phase } \\
\text { (in seconds) }\end{array}$ & $\begin{array}{l}\text { Distance Traveled: } \\
\text { Search Phase } \\
\text { (in meters) }\end{array}$ \\
\hline Normal view & $4.13(1.43)$ & $4.59(1.29)$ & $513.61(217.97)$ & $489.22(194.51)$ \\
Aerial view & $4.38(1.24)$ & $5.53(.80)$ & $417.83(195.27)$ & $366.41(133.89)$ \\
No orientation cues & $4.66(1.15)$ & $5.13(1.07)$ & $452.98(207.01)$ & $425.98(172.43)$ \\
Orientation cues & $3.84(1.39)$ & $5.00(1.27)$ & $476.56(216.82)$ & $427.67(182.83)$ \\
Standard environment & $3.91(1.23)$ & $4.78(1.26)$ & $472.71(195.33)$ & $438.45(168.36)$ \\
Enhanced environment & $4.59(1.36)$ & $5.34(1.00)$ & $457.45(227.43)$ & $415.59(185.75)$ \\
\hline
\end{tabular}

traveled in meters) and the mean time (minutes) to locate the destinations.

The room placement test required participants to place labeled cutouts of the destination rooms in their correct location on a map outline of the third floor. This computer-based test required participants to click and drag each of the six destination rooms to their correct position on the map. Participants had as much time as they needed to complete the test. Measures selected for this test included distance error (the distance in meters between correct location and the placed location) and room placement time (the time in minutes required to place all six rooms on the map outline). The magnitude of room placement distance error was a function of both bearing errors and distance estimation errors.

\subsection{Results and Discussion}

\subsection{Performance during Training Phases.}

During the exploration training phase, the number of rooms that were correctly located was recorded. During the search training phase, the number of rooms located, total search time, and distance traveled were recorded. Each of these measures was normalized using the appropriate power transformation before analysis. These performance measures, along with gender, were used in a $2 \times 2 \times 2 \times 2$ MANCOVA to determine the effects of viewing perspective, environment type, orientation cues, and gender on training phase performance. The covariate, the cube comparisons test score, was computed by subtracting the number of incorrect items from the number of correct items. The untransformed means of the measures of survey learning and standard deviations (in parentheses) are presented for each level of navigation aids in table 1 .

All effects were evaluated at a $p$-level of 0.01 to determine statistical significance. There was a significant main effect for viewing perspective $(F(4,43)=6.36, p<$ $0.001)$, but not for environment type $(F(4,43)=3.09$, $p=0.025)$ or for orientation cues $(F(4,43)=2.21, p=$ $0.08)$. Gender had no effect, and there were no significant interactions. The effect of the covariate, cube comparisons score, was not significant $(F(4,43)=1.74, p=$ $0.16)$. The covariate was significantly correlated with search time $(r(63)=0.375, p<0.01)$ and distance traveled $(r(63)=0.301, p<0.01)$, during the search training phase, but not with the number of destinations found during training.

Follow-up ANOVAs revealed that having an aerial view significantly increased the average number of rooms found during the search training phase $(F(1,46)=20.59, p<0.001)$, but not during the exploration phase $(F(1,46)=0.80, p=0.38)$. Viewing from an aerial perspective decreased the distance traveled in locating destinations $(F(1,46)=13.79, p<$ $0.001)$ but did not affect the total search time 
Table 2. Mean Time Spent at Each Viewing Altitude by Training Phase

\begin{tabular}{|c|c|c|c|c|c|}
\hline \multirow[b]{2}{*}{ Training Phase } & \multicolumn{5}{|c|}{ Viewing Time (in seconds) Spent at Each Altitude } \\
\hline & $15 \mathrm{~m}$ & $30 \mathrm{~m}$ & $120 \mathrm{~m}$ & All Altitudes & Ground Level \\
\hline Free Exploration & 34.51 & 47.55 & 3.12 & 85.18 & 497.70 \\
\hline Destination Search & 31.17 & 30.86 & 0.91 & 62.94 & 354.89 \\
\hline
\end{tabular}

$(F(1,46)=5.54, p=0.023)$. None of the ANOVA interactions were significant.

Time spent in the aerial view varied among participants and across training phases. Overall, participants used the aerial view more during free exploration than during the destination search phase. (See table 2.) Time spent at different viewing heights also varied. Note that participants spent more time at the $15 \mathrm{~m}$ and $30 \mathrm{~m}$ heights than at the highest altitude. The highest altitude may have been selected less often because the time required to reach it was much longer. Participants chose the $15 \mathrm{~m}$ and $30 \mathrm{~m}$ viewing heights equally as often during destination search, but selected the $30 \mathrm{~m}$ height more often during free exploration. This suggests that the $30 \mathrm{~m}$ height was preferred for exploring the $\mathrm{VE}$ as a whole, but $15 \mathrm{~m}$ and $30 \mathrm{~m}$ heights were chosen equally when locating specific destinations.

\subsubsection{Performance on Survey Knowledge}

Tests. A $2 \times 2 \times 2 \times 2$ (perspective view by orientation cue by environment type by gender) factorial MANCOVA design was used to analyze the survey knowledge test results. The covariate was the score on the cube comparisons test. The projective convergence test dependent measures were angular heading error and distance error. A check of the mean constant (heading) error revealed it differed significantly from $0(t(63)=$ $5.08, p<0.001)$, suggesting a small clockwise pointing bias (mean constant error $=12.25$ deg.). Constant error was not significantly affected by the navigation aids, nor was it significantly correlated with absolute (heading) error. Variable (heading) error was not analyzed separately due to its extremely high correlation with absolute heading error $(r(64)=0.96)$. The dependent measures for the room placement test were distance error and placement time, and the dependent measures for the most direct route test were mean distance error and travel time. The distributions for all of the dependent measures (except heading error) were markedly nonnormal (positively skewed) and were normalized for analysis by using power transformations. The exponents for the power transforms ranged from -0.65 to 0.25 for the various dependent measures. For some measures, negative power transformation exponents resulted in directional sign changes in calculated correlation coefficients. Untransformed means and standard deviations of the dependent measures by level of navigation aid are shown in table 3 . All effects were evaluated at a $p$-level of 0.01 to determine statistical significance. There was a significant main effect for viewing perspective $(F(6,42)=3.84, p<0.01)$, but no effects for either orientation cues or environment type. There were no significant interactions. The effect of the covariate, cube comparison score, was not significant $(F(6,42)=2.28$, $p=0.054)$. The covariate was significantly correlated with search test distance error $(r(64)=-0.433, p<$ $0.001)$ and average search time $(r(64)=0.484, p<$ $0.001)$, but not with distance error $(r(64)=0.286, p=$ $0.011)$ or heading error $(r(64)=-0.259, p=0.02)$ on the projective convergence test, nor with distance error $(r=0.162, p=0.094)$ and room placement time $(r=0.166, p=0.10)$ on the room placement test.

Viewing perspective significantly affected three of the six dependent measures including projective convergence angular error $(F(1,47)=9.75, p<0.01)$, projective convergence distance error $(F(1,47)=9.75, p<$ $0.01)$, and room placement distance error $(F(1,47)=$ $9.13, p<0.01)$. No effects were found for room place- 
Table 3. Untransformed Means and Standard Deviations for Survey Knowledge Test Measures as a Function of Type of Navigation Aid

\begin{tabular}{|c|c|c|c|c|c|c|}
\hline \multirow{3}{*}{$\begin{array}{l}\text { Navigation } \\
\text { Aid Provided }\end{array}$} & \multicolumn{2}{|c|}{ Most Direct Route Test } & \multicolumn{2}{|c|}{ Projective Convergence Test } & \multicolumn{2}{|c|}{ Room Placement Test } \\
\hline & Distance & Search & Heading & Distance & Distance & Placement \\
\hline & Error $(\mathrm{m})$ & Time (sec.) & Error (deg.) & Error $(\mathrm{m})$ & Error $(\mathrm{m})$ & Time (sec.) \\
\hline Normal view & $23.99(21.38)$ & $75.77(26.81)$ & $57.8(19.52)$ & $19.91(5.74)$ & $26.33(22.31)$ & $115.19(90.61)$ \\
\hline Aerial view & $23.13(29.29)$ & $76.56(36.34)$ & $44.99(19.19)$ & $16.31(5.78)$ & $15.08(12.86)$ & $92.25(38.10)$ \\
\hline $\begin{array}{l}\text { No orientation } \\
\text { cues }\end{array}$ & $28.44(31.86)$ & $78.89(33.97)$ & $53.52(19.96)$ & $18.02(5.15)$ & $22.71(19.50)$ & $93.16(63.03)$ \\
\hline Orientation cues & $18.68(15.85)$ & $73.44(29.50)$ & $49.27(20.64)$ & $18.20(6.83)$ & $18.69(18.49)$ & $114.28(75.70)$ \\
\hline $\begin{array}{l}\text { Standard } \\
\text { environment }\end{array}$ & $19.33(17.36)$ & $69.29(24.64)$ & $51.58(21.45)$ & $18.77(7.32)$ & $21.11(20.90)$ & $103.47(76.64)$ \\
\hline $\begin{array}{l}\text { Enhanced } \\
\text { environment }\end{array}$ & $27.79(31.26)$ & $83.04(36.54)$ & $51.20(20.25)$ & $17.45(4.31)$ & $20.30(20.09)$ & $103.97(63.72)$ \\
\hline
\end{tabular}

ment time, or for either of the most direct route test measures.

Performance on the survey knowledge tests varied widely, even among participants who used the same navigation aids. This suggests large individual differences in spatial abilities of our participants. Support for this notion is found in the distribution of scores on the cube comparisons test. Scores on the test ranged from -3 to 40 with a mean score of 18.02 and a standard deviation of 10.66. Significant correlations of cube comparison test scores with destination search test search time $(r(64)=0.48, p<0.01)$ and distance error $(r(64)=-0.43, p<0.01)$ suggests that these VE survey knowledge measures vary with individual differences in spatial ability. The correlations of cube comparison scores with the other survey knowledge measures were projective convergence distance error $(r(64)=0.29$, $p=0.02)$ projective convergence heading error $(r(64)$ $=-0.26, p=0.039)$ room placement distance error $(r(64)=0.17, p=0.19)$, and room placement time $(r(64)=-0.16, p=0.20)$.

For the aerial group, Pearson correlations were computed to examine relationships between the amount of time spent in the aerial view and subsequent performance on the survey knowledge tests. A significant cor- relation was obtained between free exploration aerial time and heading error $(r(31)=-0.48, p<0.01)$. The direction of this significant correlation and correlations between aerial time and other survey knowledge measures indicated that increased aerial time during free exploration was associated with increased survey knowledge. These data suggest that during free exploration, participants were using the aerial view to locate destinations relative to one another and to investigate the layout as a whole. Significant correlations of aerial time with search distance error $(r(32)=0.56, p<0.001)$ and search time $(r(32)=-0.57, p<0.001)$ for the search training phase and the direction of these correlations indicates that increased aerial time during search was associated with poor performance. During the search task, participants likely used the aerial view as a crutch to locate a particular destination, rather than as a tool to enhance their knowledge of the building configuration. This suggests that the advantage of an aerial view in acquiring survey knowledge depends on how the participants use the aerial view.

A one-tailed $t$-test was performed to determine if the local orientation cue resulted in fewer collisions. The mean collision rate was $M=0.45$ collisions per minute, s.d. $=0.54$, for those participants who had orientation 
Table 4. Number of Participants Who Reversed Room Placement by Navigation Aid Category

\begin{tabular}{lllllll}
\hline & \multicolumn{2}{l}{ No Orientation Cues Added } & & \multicolumn{2}{l}{ Orientation Cues Added } & \\
\cline { 2 - 3 } & Normal Viewing & Aerial Viewing & & Normal Viewing & Aerial Viewing & Totals \\
\hline Standard environment & $3^{*}$ & 0 & 2 & 0 & 5 \\
Enhanced environment & 2 & 0 & 0 & & 1 & 3 \\
Totals & 5 & 0 & 2 & 1 & 8 \\
\hline
\end{tabular}

${ }^{*}$ Control group had no navigation aids.

Table 5. Number of Top Performers on Room Placement Test by Navigation Aid Category

\begin{tabular}{|c|c|c|c|c|c|}
\hline & \multicolumn{2}{|c|}{ No Orientation Cues Added } & \multicolumn{2}{|c|}{ Orientation Cues Added } & \multirow[b]{2}{*}{ Totals } \\
\hline & Normal Viewing & Aerial Viewing & Normal Viewing & Aerial Viewing & \\
\hline Standard environment & $1^{*}$ & 4 & 0 & 5 & 10 \\
\hline Enhanced environment & 1 & 0 & 2 & 3 & 6 \\
\hline Totals & 2 & 4 & 2 & 8 & 16 \\
\hline
\end{tabular}

${ }^{*}$ Control group had no navigation aids.

cues and $M=0.78$ collisions per minute, s.d. $=1.31$, for those who did not. This difference was not statistically reliable $(t(61)=1.31, p=0.10)$, however. Participants who had a higher collision rate may be less adept at using the VE walking interface. The mean collision rate for all participants completing this study was 0.61 collisions per minute with a standard deviation of 1.0. This would suggest large individual differences in proficiency in using the VE walking interface.

\subsubsection{Poorest and Top Performers on Room}

Placement Task. A small number of participants reversed the placement of rooms so that rooms located at the north end of the building were placed at the south end and vice versa. This happened despite efforts to reorient participants before testing their room placement. Reversals indicate disorientation, which can have serious practical consequences for tasks dependent on accurate spatial knowledge. Table 4 shows the number of participants in each navigation aid condition that reversed placement of the rooms. Five participants completely reversed their room placement, and three others made partial reversals. These participants tended to be among the poorest performers. Of the eight who reversed, three were in the control group, and seven had only a ground-level view; only one had an aerial view.

Table 5 shows the number of performers by navigation aid who were among the top $25 \%$ of performers on the room placement test (upper quartile) in our sample. Twelve of the sixteen top performers were in the aerial group, and only four were in the normal view group. Only six of the sixteen were in the enhanced environment, and ten were in the standard environment. Eleven of the sixteen top performers had orientation cues; five did not.

\subsubsection{Performance on the Retention Test. A} repeated-measures, mixed-model ANOVA was used to determine the effects of the navigation aids and gender on retention. The within-subjects measure was testing occasion (initial testing phase versus retention phase). A single measure of accuracy — the distance error between 
the judged room placement and the correct placement-was the dependent measure. Before analysis, the distance error scores were normalized using a power transformation. Over a one-week retention interval, performance on the room placement test did not change significantly $(F(1,42)=3.24, p=0.079)$. However, a significant retention by gender interaction suggests that females retained more knowledge of the spatial layout than did males $(F(1,42)=7.58, p<0.01)$. On the average, performance on the retention test was $3 \%$ less than on the initial room placement test. This represents a placement difference of approximately $0.74 \mathrm{~m}$. The correlation between scores on the initial test and retention test was $r(59)=0.90, p<0.001$, indicating high test-retest reliability for the room placement test.

The ANOVA revealed a significant main effect for viewing perspective on the retention test: $F(1,42)=$ $9.18, p<0.01$. Participants who had access to an aerial view during training performed better on the retention test than those who did not. There was also a significant interaction between viewing perspective and environment type $(F(1,42)=8.38, p<0.01)$. The nature of the interaction suggests that having an aerial view improved retention scores only for participants exposed to the standard environment. Participants who had both aerial views and the enhanced environment might have experienced information overload, and could not take advantage of the additional information provided by the aerial view.

3.2.5 Simulator Sickness. Simulator sickness was a major obstacle in conducting this research. Sixteen of the original 64 participants in this research (five men and eleven women) could not complete the experiment because of severe simulator sickness symptoms. The mean SSQ total score was $M=60.54$, s.d. $=32.98$ for those who withdrew. This mean simulator sickness score is nearly twice that of those who completed the experiment. To maintain a sample size of 64 participants, these participants had to be replaced by sixteen participants who were less susceptible to simulator sickness. For those who completed the experiment, the level of simulator sickness had no significant effects on measures of survey knowledge.

\section{Conclusions}

To summarize, research participants were required to learn the layout of one floor of an office building as portrayed in a virtual environment. Because virtual environments typically do not completely and accurately represent the real world, the VE was enhanced with various navigation aids to compensate for the shortcomings inherent in VEs. These included orientation cues, a themed environment enhanced with sights and sounds and divided into sectors, and the provision of an aerial perspective. The navigation aids were provided during the training, but they were not available during survey knowledge testing. Hence, the navigation aids could improve performance either during training, during testing, during both, or not at all. Navigation aids that improve spatial performance during training do not necessarily increase performance on spatial knowledge tests. To the extent that navigation aids are used as a crutch during training, no test performance gains are achieved. Darken and Goerger (1999) also observed that participants perform poorly when they use VE navigation aids as a crutch. If aids are used to structure and organize the environment, then real performance gains can be realized.

\section{I Orientation Cues}

The orientation cues did not significantly affect performance either during the training phases or during testing. Contrary to expectation, providing a global orientation cue (the flagpole) did not improve bearing estimates or the other survey knowledge measures. Its lack of effectiveness may have been due to the inability of the participants to discriminate small changes in bearing. The long, narrow building shape likely contributed to the poor bearing discrimination.

\subsection{Enhanced Environment Augmented with Images and Sounds}

The enhanced environment did not significantly alter performance in the VE either during training or on the test. In this research, participants apparently did not 
use the enhancements during training to structure the VE, despite instructions to do so. Had the enhancements been used as instructed, the result might have been different. Instead, some participants used the themed objects and associated sounds as landmarks. Although there was some indication that this strategy helped participants locate the destinations during training, the strategy was useless when these landmarks were removed for testing purposes. Participants' failure to use the enhancements was not because they could not remember the themes associated with each destination. On the most direct route test, with enhanced environment landmarks removed, participants trained in the enhanced environment recalled the themes associated with destinations almost $89 \%$ of the time. They recalled the names of the destinations only $86 \%$ of the time.

They seemed to associate the destinations with their respective theme objects, but they did not form a mental map that organized the themed quadrants relative to each other. Perhaps their workload was too great to devote the necessary cognitive resources to organize the environment into quadrants as instructed.

\subsection{Aerial Perspective View}

In contrast, the aerial view led to better spatial performance both during training and on tests of survey knowledge. Participants were better able to structure and organize their environment if they had access to aerial views. It is not simply the amount of time spent in the aerial view that seems to determine how well the building layout is learned. Rather, it is how participants use their aerial time that affects spatial learning. If aerial time is used only to locate specific destinations, little survey learning will occur, but, if it is used to explore and organize the environment, then it will increase survey learning. Viewing height is important both for recognizing landmarks and for being able to view large portions of the environment simultaneously. In this experiment, the $30 \mathrm{~m}$ viewing height seemed to provide the best combination of landmark recognition and a large-scale view. With different environments, other viewing heights may be optimal.

Being provided an aerial perspective or having suffi- cient experience with an environment and ability to generate an aerial perspective seems the key to a highly evolved state of survey knowledge. Several converging lines of evidence support this conclusion. Not only did participants with an aerial view perform better on our survey knowledge measures, they were less likely to reverse the placement of rooms in the environment and more likely to be among the top performers. Participants who were afforded an aerial view also performed more consistently across the various measures of survey knowledge. Access to aerial perspectives had significant effects on tasks that do not necessarily require adoption of a survey perspective (that is, ground-based pointing and distance estimation). This result suggests that aerial views enable more-flexible spatial behavior (Waller, 2001).

\subsection{Disorientation}

Reversals indicate an orientation problem. Knowing the location of rooms relative to one another is of little use if wayfinders head off in a direction opposite of their intended destination. Although the wayfinder may realize that he/she chose the wrong direction when the expected landmarks are not found, it still represents a serious error with obvious practical consequences.

Therefore, we treated reversals as serious errors rather than treating them as artifacts that have no bearing on survey knowledge. Providing an aerial view nearly eliminates reversals, as only one participant given an aerial view reversed any room positions.

Participants may become disoriented following a collision. Widening hallways and doorways and other changes to the VE appeared to reduce the collision rate to one-fifth of that observed in previous research (Witmer et al., 1995). The lower collision rate in this study could also be the result of using a more natural interface to control movement than was used before. The walking interface in this study was easier to use than the interface that controlled movement manually. Still, there were large individual differences in proficiency with the walking interface. Although we reduced the average collision rate relative to that obtained by Witmer et al. (1995), higher collision rates in this study were still as- 
sociated with poorer performance. Providing local orientation cues did not result in further significant reductions in collision rates, probably because collision rates were already low. The fact that the local orientation cues did not significantly reduce the rate of collisions explains in part why participants given orientation cues performed no better than did those without these cues on the survey knowledge tests.

\subsection{A Preferred Measure of Survey Knowledge}

Of the tests used to measure survey knowledge in this research, the room placement test is likely the best measure of an individual's capability to generate an aerial view. Assuming that being able to generate an aerial view represents a more evolved or advanced stage of survey knowledge, this test is a useful indicator of advanced survey knowledge. Room placement distance error was also sensitive to retention losses. This is not to say that other measures are not useful. Projective convergence measures might even be preferred for assessing performance in the earlier stages of survey knowledge before an aerial perspective has fully developed. Although performance on projective convergence measures (such as pointing and distance estimation accuracy) may benefit from having an aerial perspective, generating an aerial perspective is not required for accurate pointing and distance estimation.

\subsection{Training Transfer from the Training to the Testing Situation}

The room placement test could be considered a de facto test of transfer from VE to the real world. It was conducted outside of the VE and required participants to construct a map like one that they might construct for real-world use. If participants have acquired sufficient spatial knowledge in a VE to construct a map that accurately represents a real-world environment, they likely can apply this knowledge in performing real-world spatial tasks. In this experiment, participants who provided an aerial view were more likely to have this spatial knowledge. Although these findings suggest that trans- fer to a real-world building would likely occur, they do not conclusively establish training transfer from the VE to the real world.

\subsection{Retention}

The performance of women on the retention test was almost identical to their performance on the initial test. This finding, coupled with the small retention losses for men, suggests that much of what was learned during training in the VE was retained over a one-week retention interval. Apparently, the decay rates for survey knowledge acquired in a VE are low.

\subsection{Implications of Research Findings}

This research has shown that survey knowledge can be acquired in a complex VE in an efficient manner without relying on external training aids (such as maps and written directions). Furthermore, this knowledge can be retained over a one-week retention interval with negligible retention loss. This is especially important for rehearsing military missions or in hostage rescue situations, in which knowledge of target areas or facilities need to be retained between mission rehearsal and mission execution. Navigation aids can improve spatial knowledge acquisition in a VE, but the manner in which they are used determines their effectiveness. Trainers must ensure that soldiers use the navigation aids to structure and organize the VE to improve acquisition and retention. Trainers may also need to limit the number of aids available at any given time to preclude information overload and allow the trainees to derive maximum benefit from the aids. Future research should focus on techniques for ensuring that the navigation aids are employed in a manner that will maximize their training effectiveness.

\section{Acknowledgments}

We thank the fine people at IST for making this research possible: Kimberly Parsons directed the VE development. Chris Kachurak modeled the VE. Jason Daly developed the soft- 
ware. Glenn Martin designed a nice operator interface. Greg Shumaker developed the audio subsystem, and Jim Parsons developed user interface components. We also thank the research consortium fellows-Bob Allen, Mar Rodriguez, Jason Kring, and Amy Bolton-who spent countless hours collecting data. Thanks also to Jeff Stahl for collecting data and scoring configuration knowledge tests. Finally, we thank Dave Waller for his insightful and thorough review and the anonymous reviewers for their helpful suggestions. Opinions expressed are those of the authors and do not represent an official position of the U.S. Army or the U.S. Army Research Institute for the Behavioral and Social Sciences.

\section{References}

Appleyard, D. (1970). Styles and methods of structuring a city. Environment and Behavior, 2, 110-118.

Bailey, J. H. (1994). Spatial knowledge acquisition in a virtual environment. Unpublished doctoral dissertation, University of Central Florida, Orlando.

Bailey, J. H., \& Witmer, B. G. (1994). Learning and transfer of spatial knowledge in a virtual environment. Proceedings of the Human Factors and Ergonomics Society 38th Annual Meeting, 1158-1162.

Bliss, J. P., Tidwell, P. D., \& Guest, M. A. (1997). The effectiveness of virtual reality for administering spatial navigation training to firefighters. Presence: Teleoperators and Virtual Environments, 6(1), 73-86.

Darken, R. P., \& Goerger, S. R. (1999). The transfer of strategies from virtual to real environments: An explanation for performance differences? Proceedings for Virtual Worlds and Simulation '99, 159-164.

Darken, R. P., \& Sibert, J. L. (1993). A toolset for navigation in virtual environments. Proceedings of ACM User Interface Software and Technology, 157-165.

- (1996). Navigating large virtual spaces. International Journal of Human Computer Interaction 8(1), 49-72.

Ekstrom, R. B., French, J. W., Harman, H. H., \& Dermen, D. (1990). Manual for kit of factor-referenced cognitive tests (Office of Naval Research Contract N00014-71-C-0117). Princeton, New Jersey: Educational Testing Service.

Gillner, S., \& Mallot, H. A. (1997). Navigation and acquisition of spatial knowledge in a virtual maze. (Technical Report No. 45). Tubingen, Germany: Max-Planc-Institute fur biologische Kybernetik.

Goerger, S., Darken, R., Boyd, M., Gagnon, T., Liles, S., Sul- livan, J., \& Lawson, J. (1998). Spatial knowledge acquisition from maps and virtual environments in complex architectural spaces. Proceedings of the 16th Applied Behavioral Sciences Symposium, 6-10.

Golledge, R. G. (1987). Environmental cognition. In D. Stokols \& I. Altman (Eds.), Handbook of environmental psychology (Vol. 1, pp.131-174). New York: John Wiley and Sons.

Guest, M., Bliss, J., \& Lohmeier, J. (1997). Landmark enhancement and strategic processing: An evaluation of strategies for spatial navigation training. Perceptual and Motor Skills $85,1123-1135$.

Guest, M., and Sadowski, W. (1997, September). Landmark Recall as a Function of Quality of Interaction during Simulated Route Navigation. Poster session presented at the $\mathrm{Hu}-$ man Factors and Ergonomics Society 41st Annual Conference. Albuquerque, NM.

Just, M. A., \& Carpenter, P. A. (1985). Cognitive coordinate systems: Accounts of mental rotation and individual differences in spatial ability. Psychological Review, 92, 137-172.

Kennedy, R. S., Lane, N. E., Berbaum, K. S., \& Lilienthal, M. G. (1993). A simulator sickness questionnaire (SSQ): A new method for quantifying simulator sickness. International Journal of Aviation Psychology, 3(3), 203-220.

Koh, G., von Wiegand, T. E., Garnett, R. L., Durlach, N. I., \& Shinn-Cunningham, B. (1999). Use of virtual environments for acquiring configurational knowledge about specific real world spaces. Presence: Teleoperators and Virtual Environments 8(6), 632-656.

Lampton, D. R., \& Parsons, J. (2001). The fully immersive team training (FITT) research system: Design and implementation. Presence: Teleoperators and Virtual Environments, 10(2), 129-141.

Levine, Kurt (1951). Field theory in social science. New York: Harper Torch Books.

Lynch, K. (1960). The image of a city. Cambridge, MA: The MIT Press.

Miller, M. S., Clawson, D. M., \& Sebrechts, M. M. (1999). Long-term retention of spatial knowledge acquired in virtual reality. Proceedings of the Human Factors and Ergonomics Society 43rd Annual Meeting, 1243-1246.

Ruddle, R. A., Payne, S. J., \& Jones, D. M. (1997). Navigating buildings in desk-top virtual environments: Experimental investigations using extended navigational experience. Journal of Experimental Psychology: Applied, 3(2), 143-159. Salzman, M., Dede, C., Loftin, R. B., \& Ash, K. (1998). Using VR's frames of reference in mastering abstract informa- 
tion. Proceedings of the Third International Conference on Learning Sciences, 249-255.

Satalich, G. (1995). Navigation and wayfinding in virtual reality: Finding proper tools and cues to enhance navigational awareness. Unpublished master's thesis. University of Washington.

Siegel, A. W. (1981). The externalization of cognitive maps by children and adults: In search of ways to ask better questions. In L. S. Liben, A. H. Patterson, \& N. Newcombe (Eds.), Spatial representation and behavior across the life span: Theory and application (pp. 167-194). New York: Academic Press, Inc.

Siegel, A. W., \& White S. H. (1975). The development of spatial representations of large-scale environments. In $\mathrm{H}$. Reese (Ed.), Advances in child development and behavior (Vol. 10, pp. 10-55). New York: Academic Press, Inc.

Singer, M. J., Ehrlich, J., \& Allen, R. C. (1998). Effect of a body model on performance in a virtual environment search task (ARI Technical Report 1087). Alexandria, VA: U. S. Army Research Institute for the Behavioral and Social Sciences. (DTIC No. ADA352026)

Thorndyke, P. W., \& Hayes-Roth, B. (1982). Differences in spatial knowledge acquired from maps and navigation. Cognitive Psychology, 14, 560-589.

Tolman, E. C. (1948). Cognitive maps in rats and men. Psychological Review, 55, 189-208.

Waller, D. A. (1999). An assessment of individual differences in spatial knowledge of real and virtual environments. Unpublished doctoral dissertation, University of Washington, Seattle.

Wickens, C. D., \& Prevett, T. T. (1995). Exploring the dimensions of egocentricity in aircraft navigation displays. Journal of Experimental Psychology Applied, 1(2), 110-135.

Wilson, P. N., Foreman, N., \& Tlauka, M. (1997). Transfer of spatial information from a virtual to a real environment. Human Factors, 39(4), 526-531.

Witmer, B. G., Bailey, J. H., \& Knerr, B. W. (1995). Training dismounted soldiers in virtual environments: Route learning and transfer (ARI Technical Report 1022). Alexandria, VA: U.S. Army Research Institute for the Behavioral and Social Sciences. (DTIC No. ADA292900)

Witmer, B. G., Bailey, J. H., Knerr, B. W., \& Parsons, K. C. (1996). Virtual spaces and real world places: Transfer of route knowledge. International Journal of Human Computer Studies, 45, 413-428.

Witmer, B. G., \& Kline, P. B. (1997). Judging perceived and traversed distance in virtual environments. Presence: Teleoperators and Virtual Environments, 7(2), 144-167.

Witmer, B. G., \& Sadowski, W. J., Jr. (1998). Nonvisually guided locomotion to a previously viewed target in real and virtual environments. Human Factors, 40(3), 478-488.

Witmer, B. G., \& Singer, M. J. (1998). Measuring presence in virtual environments: A presence questionnaire. Presence: Teleoperators and Virtual Environments, 7(3), 225-240. 